\title{
Blast wave induced flows in semicircular canals
}

\author{
Mohammad Movassat ${ }^{1}$, Nasser Ashgriz ${ }^{1}$, , Bob Cheung ${ }^{2}$ \\ ${ }^{1}$ Department of Mechanical \& Industrial Engineering, University of Toronto, Toronto, ON, Canada, M5S-3G8 \\ ${ }^{2}$ DRDC, Toronto, ON, Canada
}

Email address:

Ashgriz@mie.utoronto.ca (N. Ashgriz)

\section{To cite this article:}

Mohammad Movassat, Nasser Ashgriz, Bob Cheung. Blast Wave Induced Flows in Semicircular Canals. American Journal of Bioscience and Bioengineering. Vol. 3, No. 1, 2015, pp. 1-7. doi: 10.11648/j.bio.20150301.11

\begin{abstract}
Dynamics of endolymph and cupula in a semicircular canal subject to high frequency oscillations imposed on the endolymph at the open section of the canal is investigated. Deformation of cupula inside the endolymph fluid is simulated numerically. A high frequency and high amplitude periodic oscillations may result in the formation of vortical motions inside the semicircular canals. The number of vortices increases with the oscillation frequency. As a result of asymmetry of the canal geometry, there is an asymmetry in the pressure variation across the cupula resulting in a net motion of cupula in a specific direction during each cycle. The motion of the cupula depends on the vibration amplitude and frequency.
\end{abstract}

Keywords: Semicircular Canal, Cupula, Endolymph, Numerical Simulations

\section{Introduction}

There are three semicircular canals (SCC) arranged orthogonally to each other, responsible for measurement of angular accelerations in three dimensions. Linear acceleration is measured by Utricular and Saccular Otoliths. Canals and Otoliths are connected to each other through a bony and a membranous labyrinth and are bathed in endolymph (high in $\mathrm{K}^{+}$and low in $\mathrm{Na}^{+}$concentration) that fills the membranous labyrinth. This balance system as well as the organ of Corti which is responsible for hearing are anatomically connected to each other and form the inner ear which is located in the temporal bone of the skull.

Figure 1 illustrates a schematic of a semicircular canal. The expanded portion of the canal is called the ampulla which consists of the Crista ampullaris where the sensory hair cells are embedded, and the cilia (hair) of the hair cells project into gelatinous partition called the cupula across the canal lumen.

Movement of the cupula due to fluid motion of the endolymph deflects the embedded cilia. Deflection of the cilia towards the kinocilium (the longest cilia) of each hair cell causes firing of the sensory nerve which signals the corresponding direction and magnitude of the movement to the central nervous system. As the head rotates, walls of the canal rotate causing the endolymph to move. Since the endolymph inside the canal has inertia, it does not flow simultaneously. This relative motion causes cupula deflection and transmits the amount of rotation to the brain through nerve system, which controls the balance. A periodic head oscillation can affect the endolymph motion and cupula deflection and consequently can cause problems in the balance mechanism.

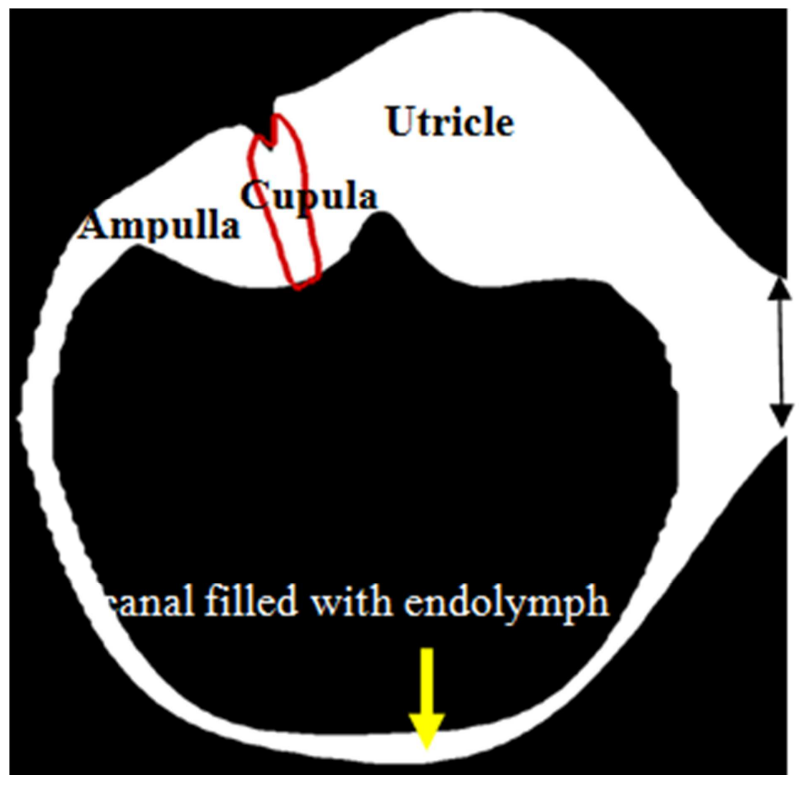

Figure 1. A two-dimensional schematic of a semi-circular canal. 
There are several models for the motion of cupula subject to a head movement. Most models are based on simplified geometries for the canal and head rotation amplitudes and frequencies within the physiological range of head motion. For instance, Steinhausen [1]modeled the canal as a torsion pendulum system, using a head rotation as a driving force for cupula, which results in the motion of endolymph, and a restoring force to attain the initial shape of cupula. By balancing these forces, and by also using a viscous damping for the endolymph, he determined the steady state deflection of cupula. Later, Vag Egmond, et al. [2] used this model to determine the resonance vibration of the torsion pendulum. They noted that the cupula motion is governed by two different time constants. Van Buskrik et al. [3] added the effect of Utricle on the torsion pendulum model.

Rabbitt and Domiano [4] developed a two-dimensional (2D) axisymmetric fluid model for the canal. They investigated the velocity distribution inside the endolymph, the pressure distribution across the cupula and the cupula deflection in response to an oscillatory motion of the canal wall. Cupula was modeled as a $2 \mathrm{D}$ elastic plate and its thickness was not considered. Also, the solution for endolymph flow was assumed to have the form of a $1 \mathrm{D}$ pipe flow. They noted that for a frequency range of $0.1-10 \mathrm{~Hz}$, the cupula displaces linearly with the forcing frequency. A later study by Damiano and Rabbitt[5] improved their previous model by assuming that the endolymph flow close to cupula deviates from the simplified 1D pipe flow. Far from the cupula, the 1D flow was shown to be valid. Multidimensionality of the flow was shown to be important when forcing frequency exceeded $1 \mathrm{~Hz}$. Frequency and amplitude of the head rotation was limited to physiological range of head motion. Also, a uniform spatial distribution for cupular deflection was assumed. Consequently, the model lacked from a morphologically accurate geometrical model of the cupula and realistic boundary conditions at the cupula endolymph and cupula-crista interfaces.

By improvements in measurement of canal geometry, the accuracy of cupula response to head rotation has been improved. In works by Ifedibaet al. [6] and Rabbitt [7],the mathematical model by Domiano and Rabbitt [5] described above was used to model more realistic three dimensional canal geometry and its effect on the development of a flow field close to the cupula and inside the canal.

In addition to the mentioned models, Computational fluid dynamics (CFD) models have been used to simulate cupula response to different actuations. Kassemi et al. [8] studied cupula deflection in response to a temperature difference actuation on the canal wall, which occurs during caloric test of the vestibular system. A finite element method was used to model endolymph motion and the response of cupula which was modeled as a deformable solid. The model was able to predict the displacement of cupula in response to temperature change of $1^{\circ} \mathrm{C}$. Details of the flow field inside the canal and cupula were obtained. In addition, Selva et al. [9] investigated the fluid interaction with cupula, and Obrist \& Hegemann [10] investigated motion of particles inside the canal.
The present work is aimed at determining dynamics of the endolymph flow and cupula subject to some extreme motions. High frequency $(>100 \mathrm{~Hz})$ and high amplitude oscillations are imposed on the canal and the movement of endolymph and cupula are investigated. Such extreme conditions may occur during the head exposure to high frequency blast waves. In addition, a realistic canal geometry and cupula shape is used to include geometrical effects on cupula deflection. A 2D two-fluid based CFD model is used to model the dynamics of cupula.

\section{Numerical Model}

A two-dimensional two fluid CFD model is used to simulate the coupled dynamics of endolymph and cupula. In this model, endolymph and cupula are considered as two immiscible fluids. Densities of endolymph and cupula are assumed to be $1000 \mathrm{Kg} / \mathrm{m}^{3}$. Endolymph and cupula viscosities are assumed to be 0.001 and 0.01 Pa.s, respectively. These properties are the same as those used by Damiano and Rabbitt [5]. Cupula is considered as a viscous fluid with deformable boundaries. The deformable interface between the endolymph and cupula are captured based on the volume of the fluid method (VOF).Both endolymph and cupula are treated as incompressible fluids. It is assumed that the temperature remains constant in the canal and it is not affected by the pressure waves.

The computational domain is shown in Figure 1, in which the black zones are solid and the white zone is the semicircular canal containing endolymph and cupula. Only one semi-circular canal is used and it is assumed that the behavior of the other canals is the same. The geometry of the canal is taken from the data provided by Curthoys and Oman [11]

The following conservation of mass and momentum equations are solved for both the endolymph and cupula:

$$
\begin{gathered}
\nabla \cdot \vec{V}=0 \\
\frac{\partial \vec{V}}{\partial t}+\vec{V} \cdot \nabla \vec{V}=-\frac{1}{\rho} \nabla p+\frac{1}{\rho} \nabla \cdot \tilde{\tau}+\frac{1}{\rho} \vec{F}_{b}
\end{gathered}
$$

Here, $\vec{V}$ is the velocity vector, $p$ the pressure, $\rho$ the density, $\tilde{\tau}$ the shear stress, and $\vec{F}_{b}$ includes any body force per unit volume. These equations are solved on a fixed Cartesian grid. In this scheme, both fluids are assumed to be incompressible. Volume of Fluid (VOF) method was employed to capture the movement of the interface between the cupula and the endolymph. In this method, avolume fraction, $C$, is defined for each computational cell, such that $C=1$ within the cells containing only the cupula, $C=0$ within cells containing only the endolymph, and $0<C<1$ within the cells having an interface between the cupula and endolymph.

As the interface between the two fluids moves, the volume fraction field is reconstructed at each time step. The interface is advected by the following equation,

$$
\frac{\partial C}{\partial t}+(\vec{V} \cdot \nabla) C=0
$$

Equations (1), (2), and (3) are discretized using a staggered 
mesh in which velocities are specified at cell faces and pressure and volume fraction at each cell center. While the scheme is three-dimensional, this work considers only one computational cell in one of the three coordinate directions, therefore, the problem is considered in two dimensions. This model has been extensively used and tested in prior literature $[12,13]$ and, therefore, more detailed discussion on the method is not provided here. A grid dependency was performed to determine optimum grid resolution for accurate simulation. Consequently, a grid with 11 grid points at the narrowest part of the cupula was found to provide grid independent results.

An oscillatory motion of the headmay cause the endolymph in the membranous labyrinth to move in an oscillatory way. Generally, such head movements result in an inertial fluid movement in SCC. However, in this work, a boundary stimulus, rather than an inertial stimulus, is considered. This type of stimulus is generated by imposing a vertical oscillatory motion at one section of the SCC: At the boundary which connects the endolymph inside the canal to other parts of the inner ear. Therefore, in the present model, the head is kept stationary and the open boundary of the SCC is oscillated. This boundary is provided with an oscillatory vertical motion with velocity amplitude of $v_{\text {wall }}$ and frequency of $f$ as:

$$
\mathrm{v}=-\mathrm{v}_{\mathrm{wall}} \times \cos (2 \pi \mathrm{ft})
$$

\section{Results and Discussion}

Effect of various high frequency and high amplitude boundary stimulus on the motion of the fluids inside the SCC is simulated. Three imposed frequencies of 100, 500, and 1000 $\mathrm{Hz}$ are used. Such frequencies are reported during head exposure to blast waves [14-17]. The velocity amplitude of oscillation is estimated based on the impact of a blast wave on a head. Once the head is impacted with a blast wave, it may move by few millimeters back and forth. A $2.5 \mathrm{~mm}$ amplitude of the motion of the head, at $500 \mathrm{~Hz}$ frequency, results in a mean velocity of $5 \mathrm{~m} / \mathrm{sec}$ for the head motion. Therefore, for a typical blast wave impact, the head may move with $5 \mathrm{~m} / \mathrm{s}$ velocity at $500 \mathrm{~Hz}$. A broad velocity amplitude range of 1$15 \mathrm{~m} / \mathrm{s}$ are considered in the present study.

Figure 2 illustrates flow streamlines for a periodic velocity boundary condition at the open end of the canal (right boundary) with a frequency of $500 \mathrm{~Hz}$ and amplitude of $5 \mathrm{~m} / \mathrm{s}$, and after 100 cycles. The streamlines are plotted at $\mathrm{t}=\mathrm{nT}+$ $\mathrm{T} / 2$ where $\mathrm{n}=0,20,40,60,80$, and 100 in Figures 2 (a)-(f), respectively. The boundary motion is initially in the downward direction. Therefore, after a quarter of a period, the streamlines indicate aclock wise (cw) flow field inside the canal. At the end of a quarter of a period, a recirculation pattern forms close to the boundary. As the boundary is moved down rapidly, not all the flow can enter into the narrow region of the canal. Therefore, part of the flow is turned upward forming a cw rotating recirculation zone after encountering the upcoming flow from the other side of the canal. During the second quarter, the wall motion is in the upward direction, and therefore, the flow inside the canal becomes counter clockwise (ccw). Close to the inlet, a second ccw rotating recirculation zone is formed. The ccw flow persist in the third quarter and reverses to $\mathrm{cw}$ in the fourth quarter of a period. The rapid up and down motion of the boundary results in the formation of series of $\mathrm{cw}$ and ccw rotating circulation zones, which interact with each other in a complex manner. Velocities are minimum at $\mathrm{t}=\mathrm{T} / 2$ and $\mathrm{t}=\mathrm{T}$ where the flow reverses. This is the time at which vortical flows inside cupula occur, which will be discussed later. Maximum velocity occurs at $\mathrm{t}=3 \mathrm{~T} / 4$ and has a magnitude of $1 \mathrm{~mm} / \mathrm{sec}$.

The flow field inside the cupula is shown in Figure 3(a)-(d) for corresponding times. It is observed that after $n=60$, vortical patterns remain almost unchanged. There are four major vortices inside the ampula and canal, two ccw and two cw. At these specific times, in addition to vortices inside the canal, there are vortices inside the cupula as well (magnified in 3); one at the middle of the cupula and one close to the Crista, where the hair cells are located. The vortical flows, where the hair cells are located, are partly because of the geometry of the canal at Crista. Results indicate that a steady circulation zones develop inside the utricle, while the flow inside the slender part of the canal reverses two times in each cycle. These vortical patterns continue until the forcing is removed and the flow field damps out.

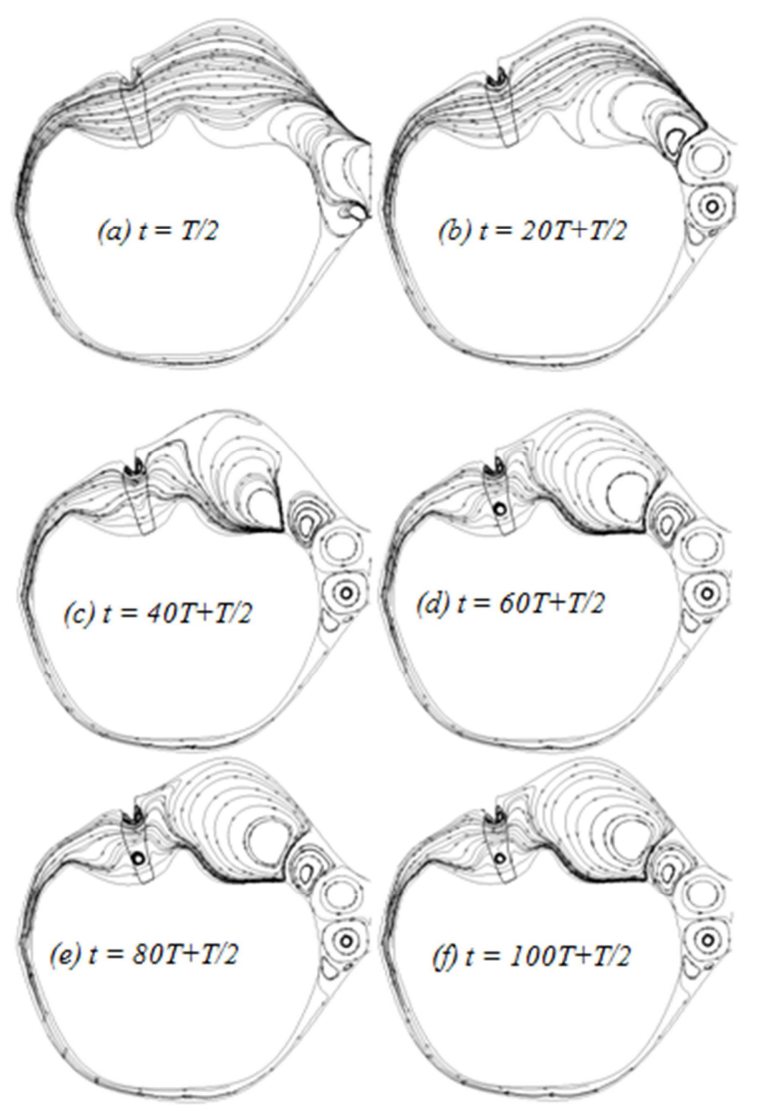

Figure 2. Streamlines at $t=n T+T / 2$, flow field inside canal for an imposed oscillation with frequency of $500 \mathrm{~Hz}$ and amplitude of $5 \mathrm{~m} / \mathrm{s}$, and after 100cycles. 
As the oscillation frequency changes, circulation patterns change as well. Figures 4 and 5 show the streamlines at $\mathrm{t}=$ $\mathrm{nT}+\mathrm{T} / 2$ for $\mathrm{n}=80$ and 100 , respectively, and for three different frequencies of $f=100,500$, and $1000 \mathrm{~Hz}$. In all of these cases, the wall velocity has been adjusted to provide 2.5 $\mathrm{mm}$ of the head motion during one cycle. As observed at500 and $1000 \mathrm{~Hz}$ cases, after 100 oscillations there are four major circulation zones, excluding those inside the cupula. Only three vortices are formed for $\mathrm{f}=100 \mathrm{~Hz}$. Steady state flow field inside cupula changes with frequency as well. At $\mathrm{f}=$ $1000 \mathrm{~Hz}$, the circulation zone in the middle of cupula is larger than those at lower frequencies.

During one cycle, the maximum velocity in the $\mathrm{cw}$ and $\mathrm{ccw}$ directions occurs at $\mathrm{t}=\mathrm{nT}+\mathrm{T} / 4$ and $\mathrm{t}=\mathrm{nT}+3 \mathrm{~T} / 4$, respectively. Figure 6 presents contours of velocity magnitude for $\mathrm{n}=60,80$, and 100 and for $\mathrm{f}=500 \mathrm{~Hz}$ and $\mathrm{v}_{\mathrm{wall}}=5 \mathrm{~m} / \mathrm{s}$. The left column is at times when the flow is cw $(\mathrm{t}=\mathrm{nT}+\mathrm{T} / 4)$, and the right column is at times when the flow is $\operatorname{ccw}(\mathrm{t}=\mathrm{nT}+3 \mathrm{~T} / 4)$. It is observed that the velocity magnitude develops and reaches a steady state after 100 cycles. Velocity magnitudes in the ccw direction are larger than those in the cw direction. This is due to the asymmetry in the canal geometry and the applied boundary condition. As a result, cupula drifts to the left during each cycle. The velocity magnitudes reveal that the average $\mathrm{cw}$ velocity field at $\mathrm{t}=100 \mathrm{~T}+\mathrm{T} / 4$, is smaller than the ccw velocity field at $\mathrm{t}=$ $100 \mathrm{~T}+3 \mathrm{~T} / 4$. Therefore, cupula has a net movement to the left during each cycle.

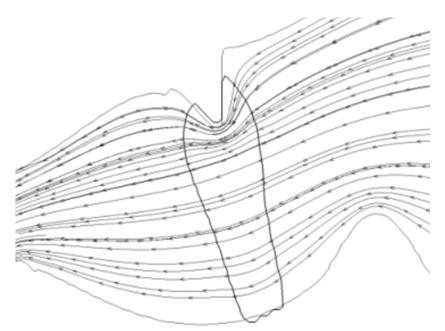

(a) $\mathrm{t}=\mathrm{T} / 2$

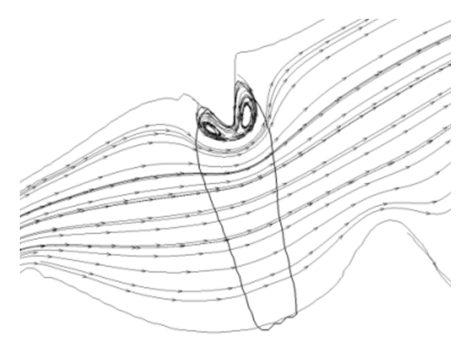

(b) $t=20 \mathrm{~T}+\mathrm{T} / 2$

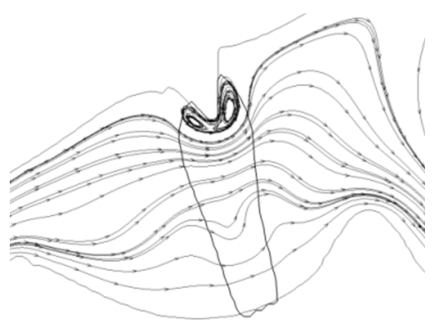

(c) $t=40 T+T / 2$

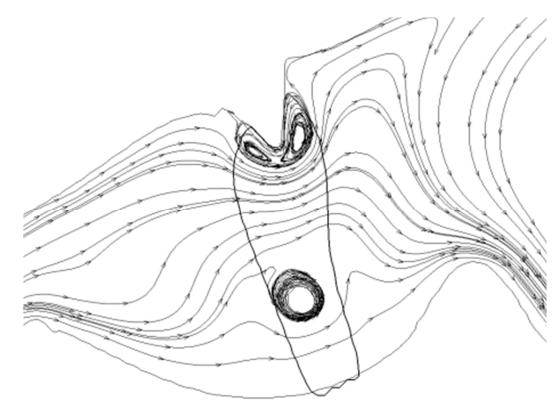

(d) $\mathrm{t}=80 \mathrm{~T}+\mathrm{T} / 2$

Figure 3. Streamlines at $t=n T+T / 2$, flow field inside the cupula for an imposed oscillation with frequency of $500 \mathrm{~Hz}$ and amplitude of $5 \mathrm{~m} / \mathrm{s}$, and after 100cycles.
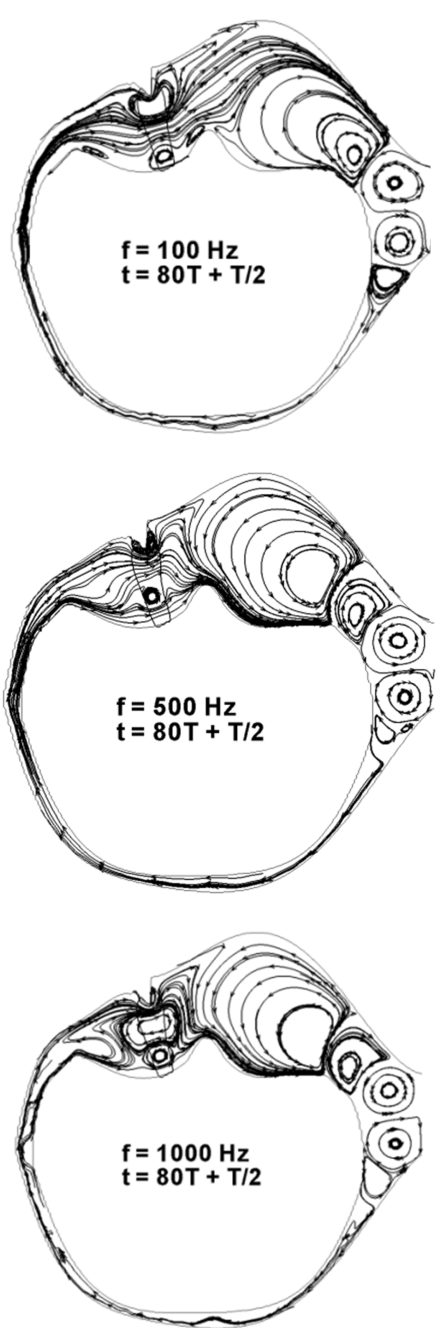

Figure 4. The effect of oscillation frequency on the vortical pattern of the flow inside the canal at 80th cycles.

Figure 7 illustrates the pressure difference across the cupula after 100 period of oscillation during one cycle. Pressure difference is calculated using pressures at two points on the surface of the cupula shown in the figure. During the first quarter $(\mathrm{t} / \mathrm{T}<100.25), \mathrm{P}_{\text {left }}$ is larger than $\mathrm{P}_{\text {right }}$ and a flow from the left to the right forms. During the second quarter $(100.25<\mathrm{t} / \mathrm{T}<100.5)$, pressure at the right side is larger than the left side and the flow from the left to the right decelerates. 
During the third quarter $(100.5<\mathrm{t} / \mathrm{T}<100.75)$, $\mathrm{P}_{\text {right }}$ is still larger than $\mathrm{P}_{\text {left }}$ and a flow from the right to the left develops. This flow field decelerates during the last quarter $(\mathrm{t} / \mathrm{T}>$ 100.75 ) by the adverse pressure gradient. During the first and the last quarters at which $\mathrm{P}_{\text {left }}>\mathrm{P}_{\text {right }}$, the maximum difference $\mathrm{P}_{\text {left }}-\mathrm{P}_{\text {right }}$ is calculated as $1 \mathrm{~Pa}$, whereas during the second and third quarters where $\mathrm{P}_{\text {left }}<\mathrm{P}_{\text {right }}$ the maximum difference is greater than $1.1 \mathrm{~Pa}$. This small asymmetry in pressure difference across the cupula during one cycle is responsible for the net motion of cupula toward the left. Pressure differences as small as $1 \mathrm{~Pa}$ across the cupula are responsible for its deformation. Mean integral of pressure force on both sides of the cupula during one cycle yields a net force from the right to the left. Pressure inside the cupula oscillates with the same frequency as the moving wall. The difference between the maximum and the minimum cupula pressure during one cycle reaches $45 \mathrm{~Pa}$.

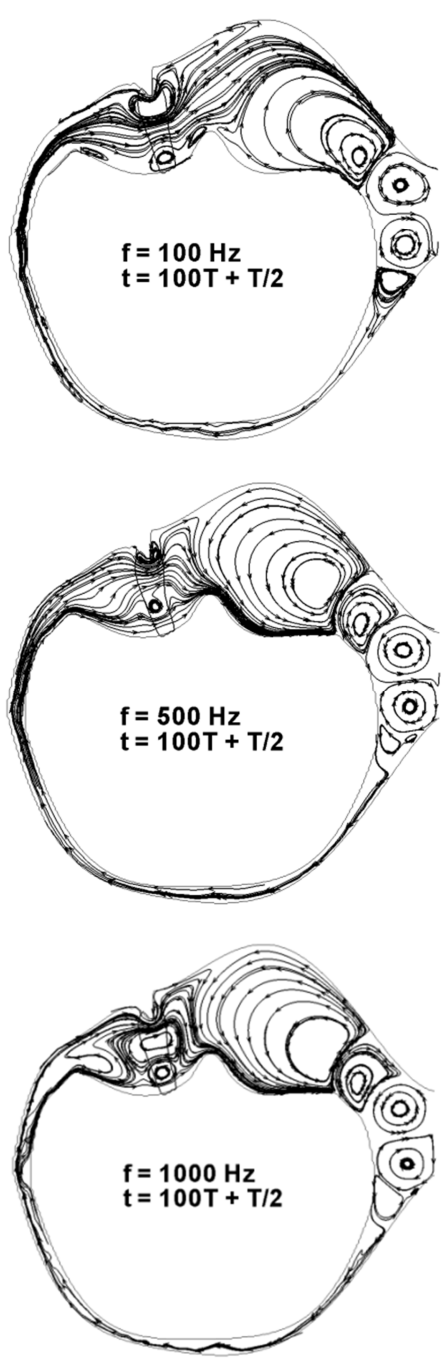

Figure 5.The effect of oscillation frequency on the vortical pattern of the flow inside the canal at 100th cycles.

The location of the center of mass of cupula in the horizontal direction versus the number of cycles of head oscillation is shown in Figure 8. During each period of oscillation, the cupula has an oscillatory motion. The initial location of the center of mass in the horizontal direction is at $\mathrm{x}=2.79 \mathrm{~mm}$ and it has a net movement towards the left side with smaller x's. Slope of this net movement can be used to determine the drift velocity of the cupula. As mentioned above, as a result of asymmetry in the pressure variation on the two sides of the cupula, which in turn results from the asymmetry in the geometry of the canal, cupula has a net motion towards the left during each period. The initial location of the cupula (in dash-dot) and its final position (solid line) are also shown on Fig. 8. The maximum displacement of the cupula measured after 150 cycles $(0.3$ seconds) is $0.043 \mathrm{~mm}$.

The cupula keeps moving to the left as long as the impact pressure continues. Figure 9 illustrates the variation of the net velocity of the cupula with frequency for four different wall velocities. This figure shows the effect of the vibration frequency and wall velocity on the net drift velocity of the cupula. Axes in this figure are in logarithmic scale. As the wall velocity increases at a certain frequency, the cupula moves faster. At a specific wall velocity, the cupula velocity decreases as frequency increases. At higher frequencies, the flow field inside the canal has less time to adjust itself to the wall velocity, therefore, the velocity of the cupula decreases. This figure only shows the mean drift velocity. As it was pointed out, internal flow fields, circulation zones, and flow configuration inside the cupula are completely different for different frequencies.

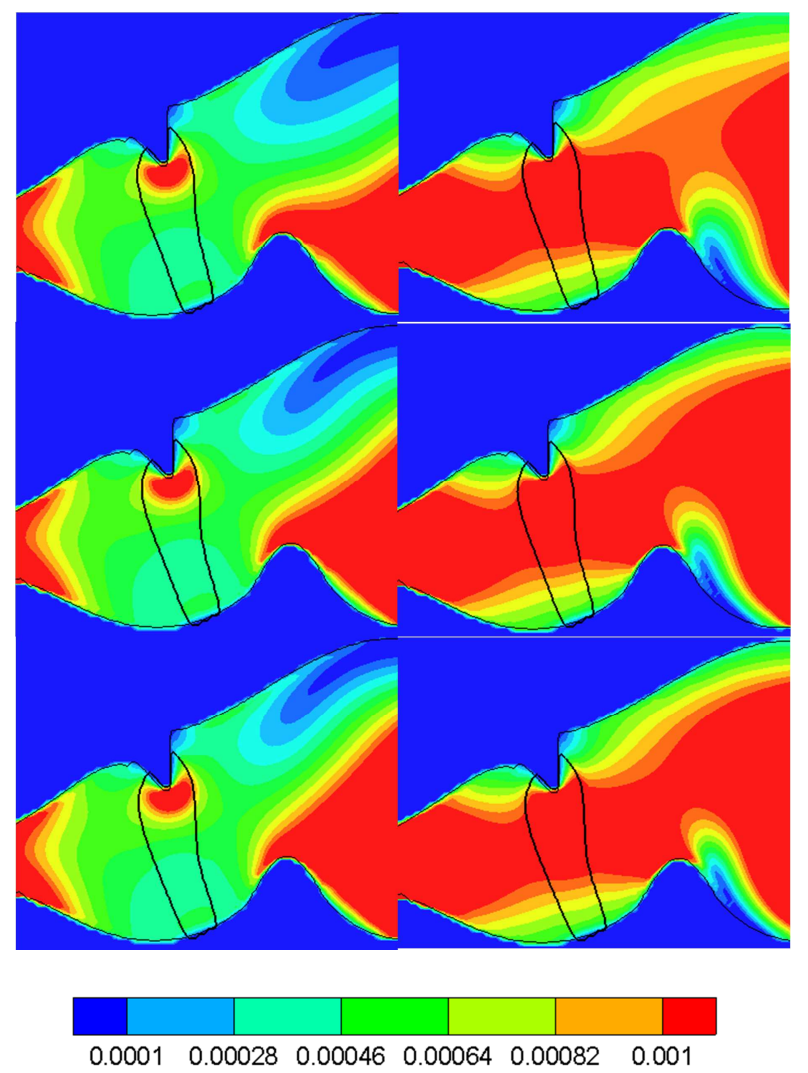

Figure 6. Velocity magnitude contour $(\mathrm{m} / \mathrm{sec})$ of Cupula at, left column: $t=$ $n T+T / 4$, right column: $t=n T+3 T / 4$. 


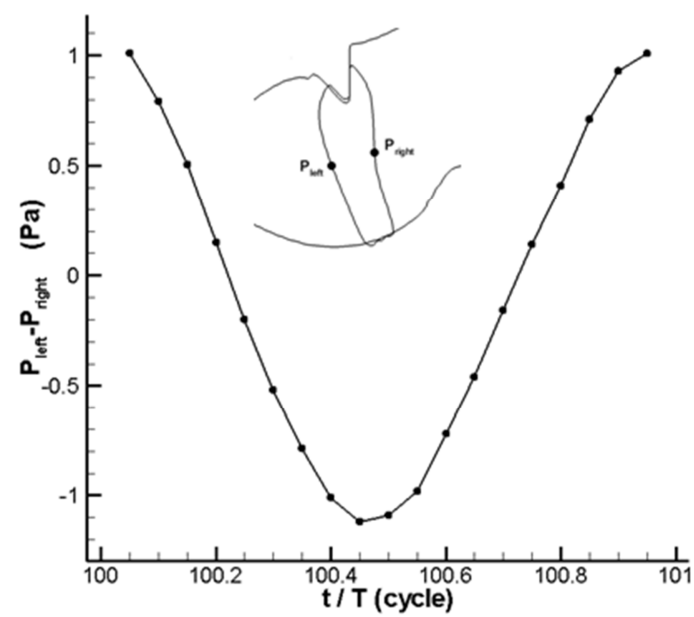

Figure 7. Pressure difference across the Cupula during one period of oscillation.

\section{Conclusion}

Effect of some extreme oscillatory movement on the flow field developed inside the semicircular canal and cupula motion is investigated. An oscillatory movement at the open section of the semicircular canal is used as the imposed oscillation. Such a motion may be observed when the canal walls or a section of the canal walls may have an oscillatory deformation due exposure to a high frequency blast wave. Although, generally, oscillation of a head may generate an inertial fluid motion inside the canal, large head impact or impacts of blast waves may result that the canal structure or a section of a canal be moved. In such cases, it is possible to have a localized region of the SCC be moved, causing a boundary stimulus on the SCC fluid rather than an inertial stimulus. The present study considers a SCC boundary stimulus for a frequency range of $100-1000 \mathrm{~Hz}$ with a amplitude range of $1-15 \mathrm{~m} / \mathrm{s}$.

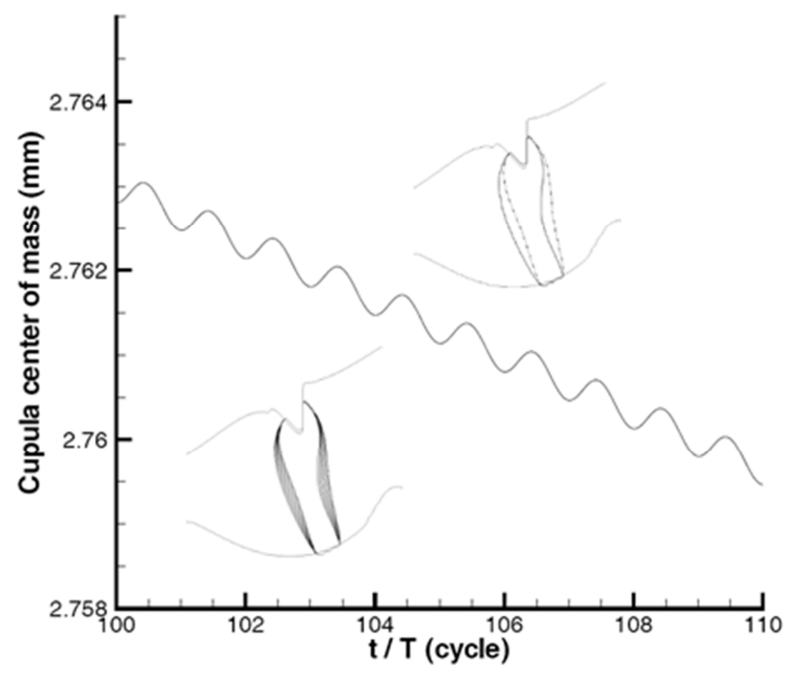

Figure 8. Location of center of mass of Cupula in the horizontal direction.

The simulation results show that a high frequency motion of the canal wall induces vortical patterns inside the canal.
These patterns form due to the asymmetric shape of the canal and the nonlinearity of the system. Once these vortices are formed, they last during the whole oscillation period. Since the semicircular canals are small and the fluids are viscous, it was surprising to see the formation of such vortical flows. However, because of the large asymmetry is the shape of the canal and the nonlinearity of the system, any movement imposed at one section of the canal would generate such vortices. The nonlinearity of the system is due to having two different fluids, endolymph and cupula, which are nonlinearly coupled, through nonlinear Navier-Stokes equations of motion. In fact, if the cupula properties were not as they are in nature (viscous gel like material), the cupula might have a chaotic behavior at certain frequencies and amplitudes. Such chaotic behavior is known characteristics of two couple nonlinear systems.

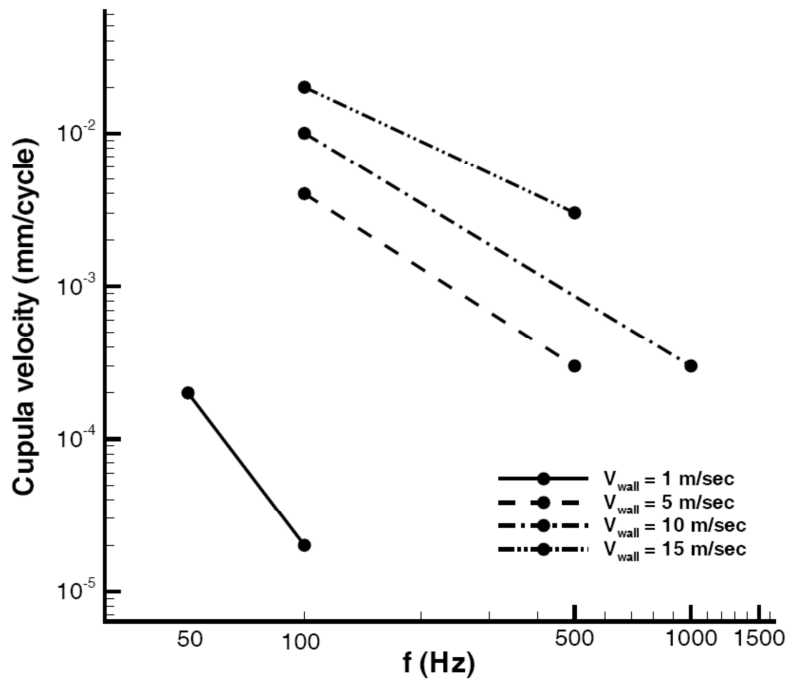

Figure 9. Cupula net velocity variation with frequency for different wall velocities.

Any movement in the endolymph may influence the cupula, and consequently a person's balance. In the present work, cupula is considered as a viscous fluid, rather than a vicious gel like material with a restoring capability. Therefore, the motion of the cupula in the present study is exaggerated, especially since the elasticity of the cupula is neglected. However, the present results have important implications in the real conditions. One important implication is the formation of an asymmetric average pressure and velocity across the cupula. Such asymmetric pressure and velocity will exist even if the elasticity of the cupula was considered. Therefore, the asymmetric pressure and velocity fields, if large enough, can cause a net drift of the cupula in one direction. If the forcing continues, this net motion of cupula may damage the balance system.

Effect of vibration frequency and velocity of moving boundary on the flow field developed inside the canal and the drift velocity of cupula are also investigated. At higher frequencies, the number of vortices inside the canal increases. Flow configuration inside cupula and close to hair cells varies with oscillation frequency as well. Increasing the 
velocity of the boundary increases the cupula drift velocity whereas increasing the vibration frequency while keeping the vibration velocity constant, decreases the cupula net velocity.

\section{References}

[1] Steinhausen, W. (1933) Uber die beobachtungen der cupula in den bognegangsampullen des labyrnithes des libendenhetchs. Pflugers Arch., 232:500-512.

[2] Van Egmond, A.A.J., Groen, J.J., \&Jongkees, L.B.W. (1949) The mechanics of the semicircular canal.J. Physiol., 110:1-17.

[3] Van Buskrik, W.C., Watts, R.G., \& Liu, Y.K. (1976) The fluid mechanics of the semicircular canals. J. Fluid Mech., 78: 8798 .

[4] Rabbitt, R.D., \& Damiano, E.R. (1992) Ahydroelastic model of macromechanics in the endolymphatic vestibular canal. $J$. Fluid Mech., 238: 337-369.

[5] Damiano, E. R., \& Rabbitt, R.D. (1996) A singular perturbation model of fluid dynamics in the vestibular semicircular canal and ampulla. J. Fluid Mech., 307: 333-372.

[6] Ifediba, M.A., Rajguru, S. M., Hullar, T.E., \& Rabitt, R. D. (2007) The role of 3-canal biomechanics in angular motion transduction by the human vestibular labyrinth. Annalas Biomedical Engineering, 35:1247-1263.

[7] Rabitt, R.D. (1999) Directional coding of three-dimensional movements by the vestibular semicircular canals.Biol. Cybern, 80:417-431.

[8] Kassemi, M., Deserranno, D., \& Oas, J.G. (2005) Fluidstructural interactions in the inner ear. Computers and Structures, 83:181-189
[9] Selva P., Oman, C.M., \& Stone, H.A. (2009) Mechanical properties and motion of the cupula of the human semicircular canal. Journal of vestibular research: Equilibrium \& Orientation 19 (3-4).

[10] Obrist, D. \& Hegemann, S. (2008) Fluid-particle dynamics in canalithiasis. J. R. Soc. Interface, 5, 1215-1229.

[11] Curthoys, I. S., \& Oman, C.M. (1987) Dimensions of horizontal semicircular duct, ampulla and utricle in the human. Acta Otolaryngol, 103:254-261.

[12] Ashgriz, N. (2011) Numerical Techniques for Simulating the Atomization Process Chapter 17 in Handbook of Atomization and Sprays, Ashgriz, N., Editor, Springer, pp: 339-35.

[13] Golpaygan, A., \& Ashgriz, N. (2008) Multiphase flow model to study channel flow dynamics of PEM fuel cell: deformation and detachment of water droplets Int. J. Comput. Fluid Dyn. 22:85-95.

[14] Taber, K. H., Warden, D. L., Hurley, R. A. (2006) Blast-related traumatic brain injury: what is known? J. Neuropsychiatry Clin. Neurosci. 18 (2), 141-145.

[15] Bauman, R. A., Ling, G., Tong, L., Januszkiewicz, A., Agoston, D., Delanerolle, N., Kim, Y., Ritzel, D., Bell, R., Ecklund, J., Armonda, R., Bandak, F., Parks, S. (2009) An introductory characterization of a combat casualty care relevant swine model of closed head injury resulting from exposure to explosive blast. J. Neurotrauma, 26 (6), 841-860.

[16] Ling, G., Bandak, F., Armonda, R., Grant, G., Ecklund, J. (2009) Explosive blast neurotrauma. J. Neurotrauma, 26 (6), 815-825.

[17] Philips, Y. Y., Mundie, T. G., Hoyt, R., Dodd, K. T. (1989) Middle ear injury in animals exposed to complex blast waves inside an armored vehicle. Annals of Otology, Rhinology and Laryngology, 98, 17-22. 